Relations industrielles

Industrial Relations

\title{
La mobilité des travailleurs urbains, par L. Hunter et G. Reid, OCDE, Paris, 1968, 239 pages.
}

\section{Pierre Dionne}

Volume 24, numéro 2, 1969

URI : https://id.erudit.org/iderudit/028035ar

DOI : https://doi.org/10.7202/028035ar

Aller au sommaire du numéro

Éditeur(s)

Département des relations industrielles de l'Université Laval

ISSN

0034-379X (imprimé)

1703-8138 (numérique)

Découvrir la revue

Citer ce compte rendu

Dionne, P. (1969). Compte rendu de [La mobilité des travailleurs urbains, par L. Hunter et G. Reid, OCDE, Paris, 1968, 239 pages.] Relations industrielles /

Industrial Relations, 24(2), 454-455. https://doi.org/10.7202/028035ar

Tous droits réservés @ C Département des relations industrielles de l'Université Laval, 1969
Ce document est protégé par la loi sur le droit d'auteur. L'utilisation des services d'Érudit (y compris la reproduction) est assujettie à sa politique d'utilisation que vous pouvez consulter en ligne.

https://apropos.erudit.org/fr/usagers/politique-dutilisation/ 
économiques, la syndicalisation, le développement des marchés et les fluctuations de salaires dans la compagnie. Ensuite, à l'intérieur même de la compagnie, les rapports statistiques indiquaient à l'auteur les rapports entre les augmentations de salaires et les accroissements de la productivité, les rapports entre la syndicalisation des ouvriers et les augmentations de salaires, rapports qu'il a su vous présenter dans les termes des théories classiques et modernes des salaires. Ainsi, dans sa conclusion, le professeur Ozanne met les salaires en rapport avec quatre grandes variables d'une théorie des salaires, soit la syndicalisation, la demande de travail sur le marché, la rigidité des échelles salariales, la productivité et le marché du produit.

Malheureusement, la valeur de ce travail est remise en question à cause de la spécificité du sujet à l'étude. En effet, si nous ne pouvons généraliser les conclusions, à quoi nous servirons les études empiriques? Le professeur Ozanne pose lui-même la question et, bien que brièvement, il $\mathrm{y}$ répond par la localisation de la compagnie ( $\ll$ urban center $)$ et par l'influence d'un syndicat (《 city-wide ») dont le champ dépassait les seuls cadres de l'entreprise. Pour maintenant, ces justifications doivent nous suffire.

Nous ne doutons pas cependant que plusieurs études de ce genre sont maintenant rendues possibles grâce aux développements de l'histoire et que leurs conclusions, comme celles de Robert Ozanne, apportent les assises empiriques et les généralisations scientifiques que nécessite la pratique des relations industrielles.

\section{René PARENTEAU}

Manpower planning for High Talent Personnel, par Eric W. Vetter, Bureau of Industrial Relations The University of Michigan, 1967, 222 pages.

La détermination des besoins en main d'oeuvre est une activité qui occupe une place importante dans la gestion rationnelle des ressources humaines. Elle fait l'objet d'une section ou d'un chapitre dans les volumes nouveaux ou dans les dernières éditions d'anciens volumes en administration du personnel au sein de l'entreprise privée.
On peut se rendre compte du nombre d'articles publiés sur ce sujet depuis le début de la présente décade en jetant un coup d'oeil sur la longue liste qui constitue l'essai bibliographique. Le volume apparaît comme un véritable effort de synthèse et de réflexion. Le but est de fournir un guide aux dirigeants et aux spécialistes de la gestion du personnel qui sont peu familiers avec la conception, la conduite et l'évaluation d'un effort de planification ou détermination des besoins de cadres supérieurs, intermédiaires et subalternes. Les premiers chapitres portent sur la nature et les phases $\mathrm{du}$ processus. On y retrouve aussi une description détaillée de la procédure à suivre et une application de cette procédure dans l'industrie de l'aéronautique. Le quatrième et le cinquième chapitres portent sur des points plus précis tels que l'établissement d'un inventaire de main d'oeuvre hautement spécialisée et la façon de mesurer la productivité. Avec le chapitre VI, on apprend à établir des prévisions en main d'oeuvre en partant du calcul du rendement anticipé. Le chapitre suivant décrit la démarche qui permet de relier les prévisions aux objectifs de rentabilité de la firme pour la période envisagée par le plan. Le préposé à la planification doit être en mesure d'évaluer les résultats des efforts déployés; il doit aussi être capable de définir sa place et son rôle au sein de l'organisation qui l'emploie. Les deux derniers chapitres traitent brièvement de ces questions.

Le dirigeant et le spécialiste du personnel doivent rendre compte que toute activité de recrutement, sélection, placement, formation, promotion devient beaucoup plus facile et efficace lorsqu'elle est précédée d'un effort de planification. C'est pourquoi, il aurait intérêt à lire rapidement ce volume qui ne présente aucune difficulté technique.

\section{Laurent BELANGER}

La mobilité des travailleurs urbains, par L. Hunter et G. Reid, OCDE, Paris, 1968, 239 pages.

A une époque où les progrès de la technologie et l'évolution des structures sont aussi rapides, la mobilité de la main-d'oeuvre devient indispensable. Si la mobilité actuelle de la main-d'oeuvre n'est pas suffisante, une intervention des 
autorités publiques pourra être nécessaire pour inciter les travailleurs à délaisser les industries et les régions en déclin pour d'autres plus favorables où il $\mathrm{y}$ a manque de main-d'oeuvre.

La présente étude, qui concerne les travailleurs urbains, fournit des informations sur le nombre et l'orientation de leurs déplacements vers de nouveaux emplois lorsqu'ils changent soit de profession, soit d'industrie, soit d'implantation géographique. Elle donne un aperç des forces qui entrent en jeu pour déterminer la direction et le choix des personnes qui acceptent ou refusent le déplacement. Elle pourra servir de base à un examen détaillé des grands problèmes que pose l'élaboration d'une politique active de la main-d'oeuvre et les conclusions qu'elle tire sont provisoires et approximatives. Enfin, cette étude énumère plusieurs éléments indispensables à la mise au point d'un système intégré.

La question fondamentale que pose cet ouvrage est de savoir si le cadre institutionnel existant aboutira à un mécanisme du marché et à une structure des salaires capables de provoquer une mobilité qui, en volume et en variété, correspondra exactement aux besoins de l'économie. Les obstacles principaux sont de caractère social et institutionnel; le manque de souplesse de la structure des salaires et l'existence de perspectives de revenus distincts des gains habituels. Les auteurs concluent néanmoins que la mobilité volontaire a plus de chance d'améliorer la répartition de la main-d'oeuvre que la mobilité involontaire.

\section{Pierre DIONNE}

The Transition from School to Work, The Princeton Manpower Symposium, May 9-10, 1968, Princeton University, New Jersey, 282 pages.

Ce volume présente dix études de base préparées pour un symposium à Princeton. Organisé conjointement par des organismes gouvernementaux et par des centres de recherches, le symposium avait pour but d'étudier le chômage chez les jeunes. En fait, il s'agissait bien plutôt d'étudier les difficultés rencontrées par les jeunes du niveau secondaire qui, n'allant pas au niveau collégial, entre après ce premier cycle d'étude sur le marché du travail. A partir de la définition du problème et de l'inventaire de ces difficultés, les participants au symposium approchent, tour à tour, les causes profondes de ce phénomène, les centres responsables et élaborent quelques solutions.

Une soixantaine d'experts se sont penchés sur le problème; ils venaient soit des milieux de l'éducation, des affaires et du gouvernement, soit des syndicats et associations. Leurs études, et spécialement celles rapportées dans ce volume, peuvent être regroupées sous trois titres principaux :

1. - les dimensions du problème du chômage chez les jeunes aux Etats-Unis;

2. - les expériences étrangères pour solutionner ce problème;

3. - les mécanismes pour aménager le passage des jeunes de l'école au travail.

Les experts n'ont pas manqué de signaler ici la responsabilité des écoles, des employeurs et du gouvernement pour prendre conscience du problème, pour chercher les solutions adéquates et les appliquer.

Il y a dans ces dix études plus que l'étude du problème, tel que ressenti aux Etats-Unis; il y a la définition de variables et facteurs clés pour approcher le problème, des méthodes de calcul pour évaluer son ampleur, des solutions propres à toute société industrielle qui fait face au chômage chez les jeunes. Nous aurions avantage à développer ces études dans le contexte québécois, à adopter les méthodes de calcul, à discuter les solutions d'un problème que nous allons rencontrer d'ici peu. En effet, au même titre que les sociétés industrielles occidentales, nous allons connaître très bientôt, à la suite de la démocratisation de l'enseignement, le problème d'intégrer au marché du travail un fort pourcentage d'étudiants qui ne sont pas aptes ou qui ne veulent pas continuer leurs études au niveau collégial ou universitaire. Il sera important, à ce moment-là, non seulement de connaître l'impact social de ce phénomène mais aussi d'avoir prévu longtemps à l'avance les mécanismes régularisateurs et le partage des responsabilités.

L. René PARENTEAU 